Disponible en ligne sur

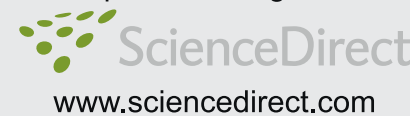

Elsevier Masson France

EM|consulte

www.em-consulte.com

\title{
Léiomyosarcome de la veine rénale gauche dans un contexte de polyarthrite rhumatoiide sous méthotrexate
}

\section{Renal leiomyosarcoma, rheumatoid arthritis and methotrexate}

\author{
R. Houitte ${ }^{a}$, Y. Jousset ${ }^{b}$, M. Delori ${ }^{a}$, P. Abgueguen ${ }^{a}$, M. Tanguy ${ }^{a}$, S. Fanello ${ }^{a, *}$
}

\author{
a Service de médecine interne et maladies infectieuses, CHU Angers, 4, rue Larrey, 49933 Angers cedex 09, France \\ b Service de chirurgie thoracique et vasculaire, CHU Angers, 4, rue Larrey, 49933 Angers cedex 09, France
}

Reçu le 14 janvier 2010 ; accepté le 27 janvier 2010

Disponible sur Internet le 26 mars 2010

\section{MOTS CLÉS \\ Léiomyosarcome ; \\ Artère rénale ; \\ Polyarthrite \\ rhumatoïde ; \\ Méthotrexate}

\section{KEYWORDS}

Leiomyosarcoma;

Rheumatoid arthritis;

Renal vein;

Methotrexate

\begin{abstract}
Résumé Le léiomyosarcome de la veine rénale est une tumeur maligne exceptionnelle dont la détection est délicate, le traitement mal codifié et le pronostic sombre. Nous décrivons le cas d'une patiente de 68 ans atteinte de polyarthrite rhumatoïde et développant un léiomyosarcome de la veine rénale gauche avec une issue fatale en moins d'un an. L'association à une polyarthrite rhumatoïde traitée par méthotrexate pose donc la question d'une combinaison fortuite ou d'un terrain favorisant compte tenu des études récentes qui tendent à prouver l'association entre ce rhumatisme et certains cancers.

(c) 2010 Elsevier Masson SAS. Tous droits réservés.

Summary Leiomyosarcoma of the renal vein is a rare and malignant tumor difficult to diagnose. No standard treatment has been defined and prognosis is poor. We described the case of a 68-year-old woman with rheumatoid arthritis treated by methotrexate who developed a leiomyosarcoma of the left renal vein with a fatal outcome in less than 1 year. Association of a leiomyosarcoma and rheumatoid arthritis raises the question of a casual association or of a predisposing factor since studies have shown increased risk of cancer with this rheumatism. (c) 2010 Elsevier Masson SAS. All rights reserved.
\end{abstract}

Le léiomyosarcome est une tumeur rare, en particulier dans sa localisation à la veine rénale avec à peine une quarantaine de cas rapportés dans la littérature. L'association à une polyarthrite rhumatoïde dans notre observation en fait

* Auteur correspondant.

Adresse e-mail : sefanello@chu-angers.fr (S. Fanello). une observation exceptionnelle mais pas unique et conduit à discuter le rôle potentiel du méthotrexate dans l'induction de ce type de tumeur.

\section{Observation}

Il s'agit d'une patiente de 68 ans hospitalisée en mars 2007 pour douleurs abdominales modérées évoluant depuis 
décembre 2006 avec apparition secondaire d'œdèmes des membres inférieurs. L'échographie, réalisée en ville, permettait d'objectiver un syndrome de masse de la veine cave inférieure.

Dans ses antécédents, on notait essentiellement une polyarthrite rhumatoïde sévère évoluant depuis 36 ans et traitée par $10 \mathrm{mg}$ de méthotrexate (depuis quatre ans) et $7 \mathrm{mg}$ de prednisone.

Le bilan biologique usuel était normal (créatininémie, bilan hépatique et numération formule sanguine) excepté une légère augmentation de l'homocystéine et la présence d'anticorps anticardiolipine (réalisés dans l'éventualité première d'une thrombose de novo).

Un scanner abdomino-pelvien, effectué en urgence, retrouvait une masse s'étendant de la veine rénale gauche jusqu'à l'auricule droite envahissant la veine cave inférieure et les veines sus-hépatiques.

Dans l'hypothèse d'une thrombose veineuse, un traitement par héparine était débuté. Un bilan d'extension était ensuite pratiqué. Le scanner thoracique retrouvait la masse intra-auriculaire sans lésion parenchymateuse pulmonaire. Le scanner cérébral était normal. Une échographie cardiaque transthoracique retrouvait une masse de $4 \mathrm{~cm}$ dans sa plus grande largeur sur $2,5 \mathrm{~cm}$, avec une bonne fraction d'éjection. Une tentative infructueuse de biopsie par voie fémorale était réalisée, conduisant à une chirurgie ; celle-ci consistait en une exérèse presque complète de la tumeur par néphrectomie gauche et cavotomie avec ouverture de l'auricule droite sous circulation extracorporelle.

L'analyse anatomopathologique de la pièce opératoire concluait à un léiomyosarcome de grade 3 . Il n'avait pas été réalisé de chimiothérapie ni de radiothérapie.

L'évolution était marquée, 11 mois plus tard, par une récidive des douleurs avec un nouveau scanner thoracique qui objectivait une récidive complète avec occlusion de la veine cave inférieure remontant jusque dans l'auricule droite, adénomégalies diffuses et des lésions nodulaires intrahépatiques (Fig. 1). La patiente devait rapidement décéder en février 2008.

\section{Discussion}

Le léiomyosarcome de la veine rénale est une tumeur maligne rare dérivée des fibres musculaires lisses de la média et touchant préférentiellement les femmes à partir de 50 ans.

La présentation clinique est classiquement insidieuse comme chez notre patiente avec des douleurs abdominales vagues ou la mise en évidence d'une masse à la palpation.

Les examens réalisés en première intention sont habituellement l'échographie et le scanner avec injection: le premier retrouve généralement une masse arrondie et hétérogène dilatant les vaisseaux; le second objective une formation à la prise de contraste hétérogène et permet de faire le bilan d'extensions local et général. Toutefois, le diagnostic est rarement fait sur l'imagerie et nécessite, soit une biopsie par voie endovasculaire, soit sur le tissu d'exérèse chirurgicale.

Le traitement recommandé est la tumorectomie complète comprenant une néphrectomie, bien que quelques cas aient été publiés avec préservation du rein

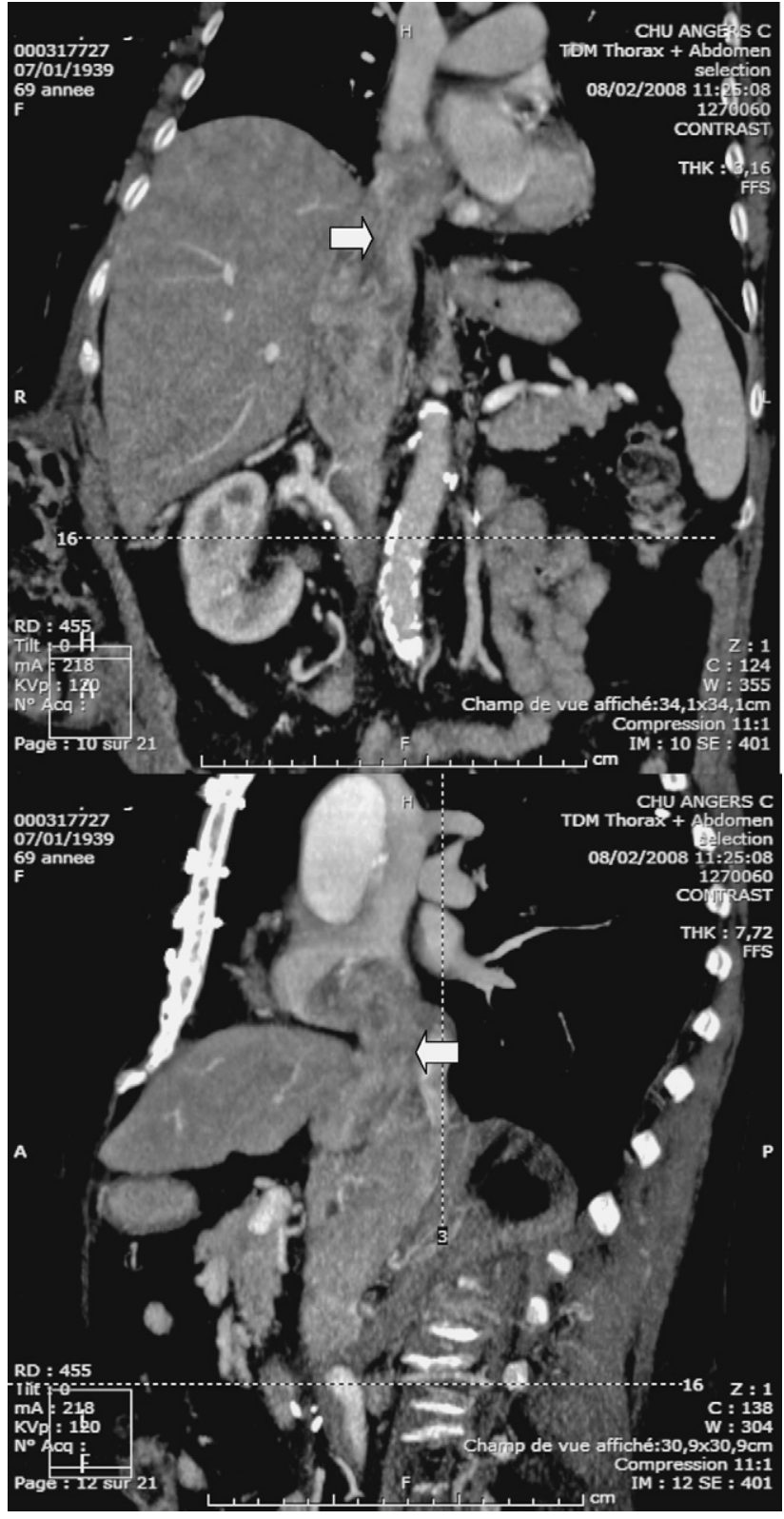

Figure 1 Aspect tomodensitométrique de la récidive tumorale envahissant la veine cave inférieure $(\rightarrow)$ avec lésions intrahépatiques.

Computed tomographic aspect of tumor recurrence spreading to the inferior vena cava $(\rightarrow)$ with intrahepatic lesions.

$[1,2]$. La chirurgie est suivie classiquement d'une chimiothérapie et/ou d'une radiothérapie. Cependant, la prise en charge thérapeutique de ce type de tumeur exceptionnelle est plutôt hétérogène [3].

L'évolution est marquée par la fréquence des métastases (50\%) touchant poumons et foie avec une atteinte ganglionnaire rare. Le taux de récurrence locale atteint les $40 \%$. La survie est dépendante du niveau de résection de la tumeur et du grade histologique. La mortalité à six ans atteint les $40 \%$.

La polyarthrite rhumatoïde est connue pour être une maladie, en particulier sous méthotrexate, favorisant les lymphomes et les mélanomes [4,5]. L'association entre léiomyosarcome, polyarthrite rhumatoïde et méthotrexate a 
déjà été évoquée en 2001 [6]. Il s'agit probablement d'une association fortuite puisque les deux pathologies partagent la même sociologie et que la polyarthrite rhumatoïde représente le rhumatisme inflammatoire le plus fréquent avec une prévalence de $0,3 \%$ en France ; mais la découverte de nouveaux cas devrait conduire à faire une analyse approfondie.

\section{Conflit d'intérêt}

Aucun.

\section{Références}

[1] Aguilar IC, Benavente VA, Pow-Sang MR, Morante CM, Meza L, Destefano V. Leiomyosarcoma of the renal vein: case report and review of the literature. Urol Oncol 2005;23:22-6.
[2] Kołodziejski LS, Dyczek ST, Gruchata A, Darasz Z, Marczyk E. Resection of the renal vein leiomyosarcoma with preservation of the kidney. Przegl Lek 2004;6:202-4 [Polish].

[3] Ceyhan M, Danacý M, Elmalý M, Özmen Z. Leiomyosarcoma of the inferior vena cava. Diagn Interv Radiol 2007;13:1403.

[4] Buchbinder R, Barber M, Heuzenroeder L, Wluka AE, Giles G, Hall $\mathrm{S}$. Incidence of melanoma and other malignancies among rheumatoid arthritis patients treated with methotrexate. Arthritis Rheum 2008;59:794-9.

[5] Franklin J, Lunt M, Bunn D, Symmons D, Silman A. Incidence of lymphoma in a large primary care derived cohort of cases of inflammatory polyarthritis. Ann Rheum Dis 2006;65:61722.

[6] Hannequin JR, Nonnenmacher J, Offner C, Simon P. Leiomyosarcoma, rheumatoid arthritis and methotrexate: an association? Joint Bone Spine 2001;68:445. 